pretreated vs -untreated WB, suggesting a production/activation of (potentially leukemia-specific) cells after kit-stimulation. Moreover kit-pretreated WB regularly and significantly improved provision, activation as well as antileukemic and leukemia-specifically directed immune reactive cells after MLC. TEM showed exosome-like structures with a typically cupshaped appearance without any differences between healthy and AML samples. fNTA revealed average vesicle sizes of 177 $\pm 23 \mathrm{~nm}$ (healthy) and 178 $\pm 17 \mathrm{~nm}$ (AML). Higher levels of EVs were detectable in AML samples compared to healthy controls in serum and after DC-culture, but lower levels after MLC independent of culture conditions.Interestingly, the number of EVs increased during cultivation of DC of AML and healthy samples, but not in AML-derived MLC samples.

Conclusions We will provide data in AML patients and healthy volunteers about a potential role of DCs- and MLCderived exosomes as biomarkers in immune responses, malignant progression or as potential therapeutic targets for AML patients.

Disclosure Information L. li: None. V. Mussack: None. E. Pepeldjiyska: None. A. Hartz: None. A. Rank: None. C. Schmid: None. E. Özkaya: None. S. Ugur: None. M. Pfaffl: None. H. Schmetzer: None.

\section{P01.12 IMPACT OF COMPLEMENTARY SUBSTANCES ON IMMUNE CELL ACTIVITY}

M Luzbetak*, N Süßenguth, E Kronemeyer, H Widera, J Werner, B Mayer. Department of General, Visceral and Transplant Surgery, LMU, Munich, Germany

\subsection{6/jitc-2020-ITOC7.25}

Background Natural substances and micronutrients are more and more included in anti-cancer therapy. However, benefit and harm both are reported from one and the same substance. This emphasizes the urgent need for the systematic analysis of a personalized approach which patient will profit from which substance.

Materials and Methods Risk analysis was performed using PBMCs isolated from patients diagnosed with advanced solid cancer. Benefit was analyzed using 3D-microtumors directly prepared from individual patient tumors. Blood cells and cancer cells were treated with different natural substances, namely curcumin, artesunate and vitamine C, as single agents and in combination therapy with guideline-directed drugs for $72 \mathrm{~h}$. Impact on cell metabolic activity was measured with the CellTiter Glo assay. The cell phenotype was described by FACS analysis.

Results In $80 \%$ of the patients natural substances induced a slight (mean: 10.7\%, range: $2.3-17.7 \%$ ) metabolic inhibition of the immune cells, which was minor in comparison to the strong immunotoxicity of chemotherapeutic drugs (e.g. 5-FU, mean: 33.5\%; Gemcitabine: 67.2\%). Contrary, $20 \%$ of the patients revealed a stimulatory effect on PBMC depending on the basic activity and the exhaustion of the immune cells. Combination therapy revealed that natural substances were able to reduce (mean: $16.4 \%$, range: $5.2-42.8 \%$ ) immunotoxicity mediated by chemotherapy. Analysis of the 3D-microtumors indicated that natural substances can mediate an anticancer effect, which was most obvious in relapsed tumors heavily pretreated with chemotherapeutic drugs. In addition, natural substances were identified as chemosensitizer. For example, curcumin was found to increase efficacy of
Mitomycin $\mathrm{C}$ in breast cancer, Bicalutamid in prostate cancer and 5-FU combined with Cisplatin in gastric cancer.

Conclusions Complementary substances have a different effect depending on dosing, timing, cell type and cell characteristics. Therefore preclinical testing is required to identify the most effective complementary substances for the individual cancer patient analyzing both immune cells and cancer cells.

Disclosure Information M. Luzbetak: None. N. Süßenguth: None. E. Kronemeyer: None. H. Widera: None. J. Werner: None. B. Mayer: None.

\section{P01.13 MERTK SIGNALING IS CRITICAL FOR T CELL PROLIFERATION AND MEMORY}

RM Powell*, MJW Peeters, A Rachbech, PT Straten. National Center for Cancer Immune Therapy, Herlev, Denmark

\subsection{6/jitc-2020-ITOC7.26}

Background Overexpression of TAM receptors, including MERTK, in some cancers are integral for chemoresistance, proliferation and metastasis. ${ }^{1}$ Our group has previously demonstrated that $\mathrm{T}$ cells also express MERTK and engagement of MERTK signaling is responsible for increased proliferation, functional capacity and metabolic fitness. ${ }^{2}$ It is therefore important to further study the effect of MERTK inhibition on $\mathrm{T}$ cell function in the context of cancer treatments where MERTK inhibitors may play a role. Here we provide evidence that MERTK inhibition impacts greatly on T cell proliferation, specifically reducing phosphorylated mTOR. We have also demonstrated that MERTK expression is increased on CD8 central memory subsets during longterm expansion providing evidence that this signaling pathway may be important for sustaining $\mathrm{T}$ memory responses.

Materials and Methods Flow cytometric analysis was used to investigate the effect of titration of MERTK small molecule inhibitor UNC2025 on healthy donor T cells activated with CD3/CD28 dynabeads. Cell trace dye was used to track proliferation of CD4 and CD8 T cells along with markers of memory differentiation (CCR7 and CD45RO), activation (CD137) and function (IFNy, Tnfa and IL-2). MERTK signaling was assessed using phospho flow cytometric methodology of phosphorylated mTOR, AKT, ERK1/2, p38-MAPK and STAT5. Long term cultures of donor $\mathrm{T}$ cells of up to 28 days were investigated for MERTK expression alongside memory differentiation.

Results We demonstrated that moderate concentrations of MERTK inhibitor reduced proliferation of activated $\mathrm{T}$ cells. Despite inhibition of cell division, cell size still increased 2 fold compared to resting cells and cell viability remained unchanged. Additionally, the proportion of central memory to effector memory populations and intracellular cytokine production was not impacted. Analysis of molecules involved in MERTK signaling revealed that phosphorylated mTOR was significantly modulated following the addition of MERTK inhibitor. Long term culture of CD8 T cells demonstrated MERTK was significantly increased following early and late re-stimulation, and expression of MERTK was strongly associated with central memory subsets.

Conclusions Our results demonstrate that inhibition of MERTK signaling on $\mathrm{T}$ cells reduces cell division where mTOR is significantly impacted. Despite this, other functional aspects, such as intracellular cytokine production remain 